This item was submitted to Loughborough's Research Repository by the author.

Items in Figshare are protected by copyright, with all rights reserved, unless otherwise indicated.

\title{
Quality assessment for virtual reality technology based on real scene
}

PLEASE CITE THE PUBLISHED VERSION

http://dx.doi.org/10.1007/s00521-016-2828-0

\section{PUBLISHER}

() The Natural Computing Applications Forum. Published by Springer

\section{VERSION}

AM (Accepted Manuscript)

\section{PUBLISHER STATEMENT}

This work is made available according to the conditions of the Creative Commons Attribution-NonCommercialNoDerivatives 4.0 International (CC BY-NC-ND 4.0) licence. Full details of this licence are available at: https://creativecommons.org/licenses/by-nc-nd/4.0/

\section{LICENCE}

CC BY-NC-ND 4.0

\section{REPOSITORY RECORD}

Jiang, Bin, Jiachen Yang, Na Jiang, Zhihan Lv, and Qinggang Meng. 2019. "Quality Assessment for Virtual Reality Technology Based on Real Scene". figshare. https://hdl.handle.net/2134/24512. 


\title{
Quality Assessment for Virtual Reality Technology Based on Real Scene
}

\author{
Bin Jiang • Jiachen Yang • Na Jiang • \\ Zhihan Lv • Qinggang Meng
}

Received: date / Accepted: date

\begin{abstract}
Virtual reality technology is a new display technology, which provides users with real viewing experience. As known, most of the virtual reality display through stereoscopic images. However, image quality will be influenced by the collection, storage and transmission process . If the stereoscopic image quality in the virtual reality technology is seriously damaged, the user will feel uncomfortable, and this can even cause healthy problems.In this paper, we establish a set of accurate and effective evaluation for the virtual reality. In the preprocessing, we segment the original reference and distorted image into binocular regions and monocular regions. Then, the IW-SSIM(Information weighted-SSIM) or IW-PSNR(Information weighted-PSNR) values over the monocular regions are applied to obtain the IW-score.At the same time, the SW-SSIM(Stereo weighted-SSIM)or SW-PSNR(Stereo weighted-PSNR) can be used to caculate the SW-score. Finally, we pool the stereoscopic images score by combing the IW-score and SW-score. Experiments show that our method is very consistent with human subjective judgment standard in evaluation of virtual reality technology.
\end{abstract}

Keywords Virtual reality · Stereoscopic images quality · Binocular regions · Monocular regions · Information weighted $\cdot$ Stereo weighted

Bin Jiang · Jiachen Yang

School of Electronic Information Engineering, Tianjin University, Tianjin, P.R. China

Na Jiang

Management services company of the first mining area, Da Gang Oilfield, PetroChina, Tianjin, P.R. China

Zhihan Lv (凶)

Dept. of Computer Science, University College London, London, WC1E 6EA, UK

E-mail: Z.Lu@cs.ucl.ac.uk

Qinggang Meng

Department of Computer Science, School of Science at Loughborough University, UK 


\section{Introduction}

Virtual reality(VR) technology is a way to create and experience the virtual world. Computer system generate a simulation environment which is a fusion of multi-source information.Users can experence the interactive operation just like in the real world $[4,5]$.

Virtual reality technology mainly includes simulation environment, perception, natural skills and sensor design. The simulation environment is produced by real-time dynamic 3D stereo images [6,7]. In addition to the computer graphics technology generated by the visual perception, there are hearing, touch, force perception, motion and other sensing $[8,9]$. The sense of smell and taste can also be produced. Natural skill refers to the rotation of the head, eyes, gestures or other human behavior action. The user's input makes realtime response[12,13]. Further more, the sensing device is a three-dimensional interactive device, which is the content we will discuss in this paper $[6,7]$.

The most acceptable information in a human sense organ is visual through our eyes[10,11]. Both in the production and life, people need more and more rich visual information to enrich our world and life[9,11]. As known, the real world is 3D three-dimensional scene. However, the majority of existing 3D stereo display device can only display 2D information, which can not give people a deep feeling $[8,9]$. In order to show the scene and the object with a deep feeling (that is, 3D), people attempt to researche on all aspects [12]. $3 \mathrm{D}$ stereo display technology research has experienced more than ten years of development and fruitful achievements have been made. 3D stereo glasses and head display equipments are used for 3D stereo display.

For the display of the world development , 3D has become a well deserved key word. TV display and the other display devices have all launched the 3D stereo display device[6]. Further more, virtual reality has become the next focus of the display device. Although many people have access to 3D information, most of them do not know the specific process.In order to answer these questions, a comprehensive understanding system is needed,which is shown in Fig. 1.

As known, Most of the virtual reality display [13] through the way of stereo images, which is shown in Fig. 1. However, the collection, storage and transmission process of stereoscopic images quality will be influenced in a certain degree[1-3].If the stereoscopic image quality in virtual reality is seriously damaged, the user will feel uncomfortable, and even cause health problems. So the stereoscopic image quality is the key index to evaluate the quality of a virtual reality system $[6]$.

Quality assessment of stereoscopic image can be divided into subjective and objective quality evaluation[14,15]. Subjective quality evaluation method allow observers to give quality score of test according to subjective perception $[25,26]$. Although its score is closer to the true visual perception, it wastes time and human resources. Objective quality evaluation method constructs a model calculats image quality by means of mathematical formula [29-33]. Its advantage is the fast speed, low cost, easy to implement, so it is more 


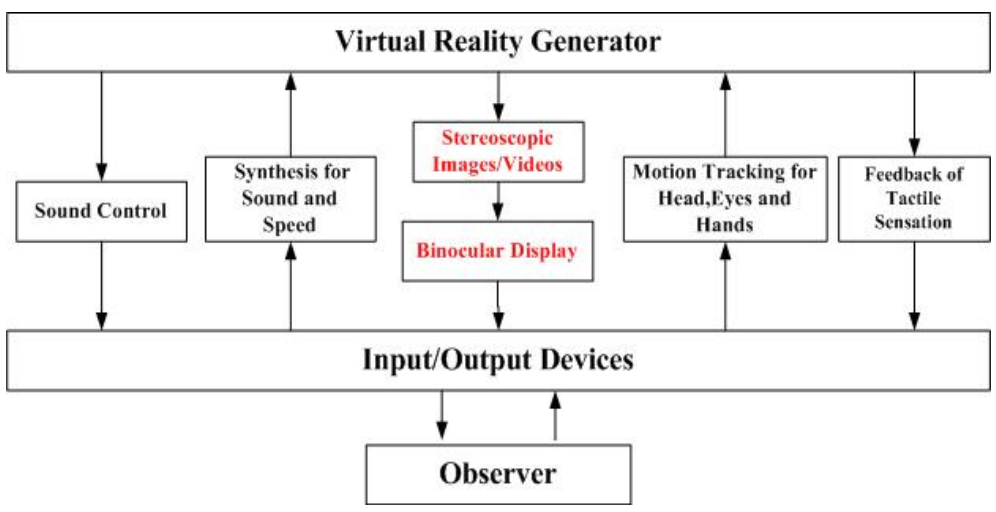

Fig. 1: The position of stereoscopic image quality in virtual reality, the red part is directly related to the content of this paper.

practical $[44,45]$. At present, there are a lot of researches on the objective evaluation of stereo image quality[17-19]. Some papers construct the models of human visual system (HVS), which can evaluate the quality of the stereoscopic image[43]. For example, the work $[22,24]$ can evaluate the quality of the stereoscopic image by the combination of the absolute difference of the left and right views on the stereoscopic perception; The other literatures use error calculation (PSNR) as a index to evaluate the quality of the stereoscopic image by simulate multi-channel decomposition characteristics[46, 47]. However, At present, the human understanding of HVS is very limited, so some literature use a simple square error algorithm mean, such as the use of the literature in[37] using PSNR method to define index of Stereo stereoscopic quality objective evaluation from image quality and sense of three-dimensional. In addition, SSIM (Structural Similarity, SSIM) algorithm [27,28] is simple and efficient, and the performance is better than PSNR and some evaluation methods such as HVS. So some literatures use the classic SSIM algorithm [40-42].

The rest of this paper is organized as follows. Sect.2 describes the background and motivation for the idea. Sect.3 makes a proposed stereoscopic image quality assessment algorithm in detail. Sect. 4 shows the experimental results . Sect. 5 concludes the paper.

\section{Background and motivation}

\subsection{Content-weighted}

Most of the image quality assessment algorithms focus on the overall information of the image, while ignoring the difference of local information. Especially in the process of stereo image processing and evaluation, local high-frequency information may have a great impact on the overall sense of stereo and comfort. Since there is less research in this area, we focus on this direction. The 

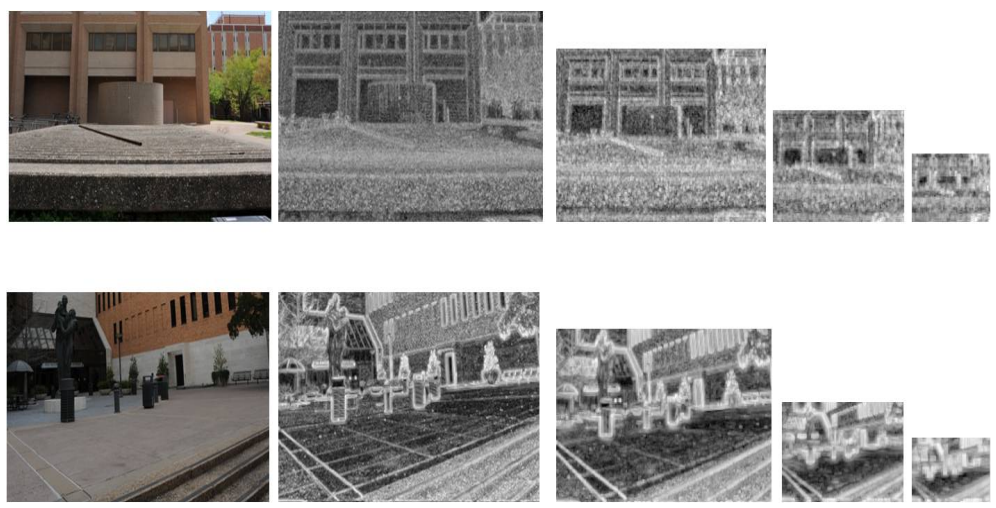

Fig. 2: The contet-weighted images for different scales,we select two left image from the LIVEI stereoscopic images databases randomly

saliency region of the image is the main signal of human visual reception, for example, the strong edge occupies a large proportion of the image information, and it is very important in the visual area [34-38].

In the existing research work, there are many methods about pooling, which can be divided into Minkowski pooling, Local distortion-based pooling ,Saliency-based pooling, Object-based pooling and so on[16,48].

These methods have achieved certain results. However, due to the sensitivity of the database, the pooling method has a dependence on the computational model. And the content of the image is not taken into account .

As a very famous theory, the problem can be well explained by GSM model[16], as described in Eq. 1,

$$
R=s U
$$

Among them, $R$ can represent the transform domain vector group of the reference image, $U$ is a zero-mean Gaussian vector,and s can be regarded as a mixing multiplier.Similiarly,it can also be used to represent the distorted image in Eq. 2,

$$
D=g R+V
$$

where $g$ is a gain factor and $V$ is additive Gaussian noise.After that, the reference and distorted images pass through a process, and we can know the perceive signals in Eqs. 3 and 4.

$$
\begin{gathered}
E=s U+N_{1} \\
F=g s U+V+N_{2}
\end{gathered}
$$


where $N_{1}$ and $N_{2}$ are the Gaussian noise just like $V$. Based on the theory in $[25]$, we can obtain the nutual information $I(R ; E)$ and $I(D, F)$. At last ,we can know the information weighted map through Eqs. 5.

$$
\omega=I(R, E)+I(D, F)-I(E, F)
$$

In the content-weighted IQA, we solve the evaluation problem from image detail information, as shown in Fig. 2. Just like the 2D image evaluation, it is generally considered that visual system Human is an optimized information feature extraction process. When using computational simulation of this process, we assume the region which contains more information in the image will cause much more attention from the observer. Specific to the pixel level, local information content can be used as the weight of the image quality assessment. In stereo image evaluation, the contribution of different regions to the three dimensional degree will be different,which will be discussed in Sect. 3. So it is necessary to divide the stereo image into binocular correlation region and monocular region.

\subsection{Structural similarity}

This is a ususl method used to evaluate the quality of the 2D image. Because it is very easy for the human vision to draw out the structure information from the image, the similarity of the structure information between the two images can be used to detect the image quality[27].

First structural information should not be influenced by the lighting effects, so it is needed to remove the brightness information in the calculation of structural information. secondly, structural information should be subject to the influence of image contrast, the computational structure information need the difference of the normalized images. Finally we can simply calculate the correlation coefficient of the two pictures after processing the image for the image structure information[28].

However, the image quality is the restriction of brightness and contrast information. so in the calculation of the image quality, information structure also need to consider the influence. We can commonly use calculation method as Eq. 6,

$$
\operatorname{SSIM}(x, y)=[l(x, y)]^{\alpha}[c(x, y)]^{\beta}[s(x, y)]^{\gamma}
$$

These three parts can be calculated separately in Eq. 7,8 and 9,

$$
\begin{gathered}
l(x, y)=\frac{2 \mu_{x} \mu_{y}+C_{1}}{\mu_{x}^{2}+\mu_{y}^{2}+C_{1}} \\
c(x, y)=\frac{2 \delta_{x} \delta_{y}+C_{2}}{\delta_{x}^{2}+\delta_{y}^{2}+C_{2}} \\
s(x, y)=\frac{\delta_{x y}+C_{3}}{\delta_{x} \delta_{y}+C_{3}}
\end{gathered}
$$


where $l(x, y), c(x, y), s(x, y)$ respectively the image brightness function, contrast function and structural similarity function of the reference images and the distortion images. $C_{1}, C_{2}$ and $C_{3}$ are very small, mainly used for prevent the denominator is zero and instability caused by the phenomenon, $C_{1}=\left(k_{1} L\right)^{2}, C_{2}=$ $\left(k_{2} L\right)^{2}, C_{3}=\left(C_{2} / 2\right)^{2}, k_{1}, k_{2}$ are far less than the normal number of $1, L$ is the value of the pixel on the way the range. $\alpha, \beta$ and $\gamma$ is parameter to control the weight of three functions, which is greater than 0 , generally, $\alpha=\beta=\gamma=1$. $\mu_{x}$, $\mu_{y}$ are the mean of $x$ and $y, \delta_{x}, \delta_{y}$ are the variance of $x$ and $y, \delta_{x y}$ is the covariance of $x$ and $y$.We can caculate them in Eq. 10,11 and 12 .

$$
\begin{gathered}
\mu_{x}=\sum_{i=1}^{N} \omega_{i} x_{i} \\
\delta_{x}=\left(\sum_{i=1}^{N} \omega_{i}\left(x_{i}-\mu_{i}\right)^{2}\right)^{1 / 2} \\
\delta_{x y}=\sum_{i=1}^{N} \omega_{i}\left(x_{i}-\mu_{x}\right)\left(y_{i}-\mu_{x}\right)
\end{gathered}
$$

In the end, we can get the expression of SSIM in Eq. 13.

$$
\operatorname{SSIM}(x, y)=\frac{1}{M} \sum_{i=1}^{M} \operatorname{SSIM}\left(x_{i}, y_{i}\right)
$$

\subsection{Binocular vision}

Binocular stereo vision is an important form of machine vision, it is based on the principle of combination for two images from different eyes. Through the position deviation of calculated image corresponding points , we can obtain geometrical information of 3D objects[18]. Image fusion of two eyes is obtained and the difference between them is observed, so that we can get a sense of the apparent depth, which establish the correspondence between features.The distance difference between the same pixel in the left and right views shows the difference between the two eyes. And we called the difference parallax (disparity) image[17].

Binocular vision contains a large amount of information, so it is necessary to map the common information in an image which can reflect left image and right image. The appearance of the cyclopean map has solved this problem[19, $20]$.

The pioneering work of binocular stereo vision began in the 60's of the last century by Roberts at the Massachusetts Institute of Technology (MIT). Masking of distorted stereoscopic images and binocular rivalry are important in binocular vision. So simulating the true cyclopean image reflect it perfectly by accounting for the display geometry,the presumed fixation,vergence and accommodation. 

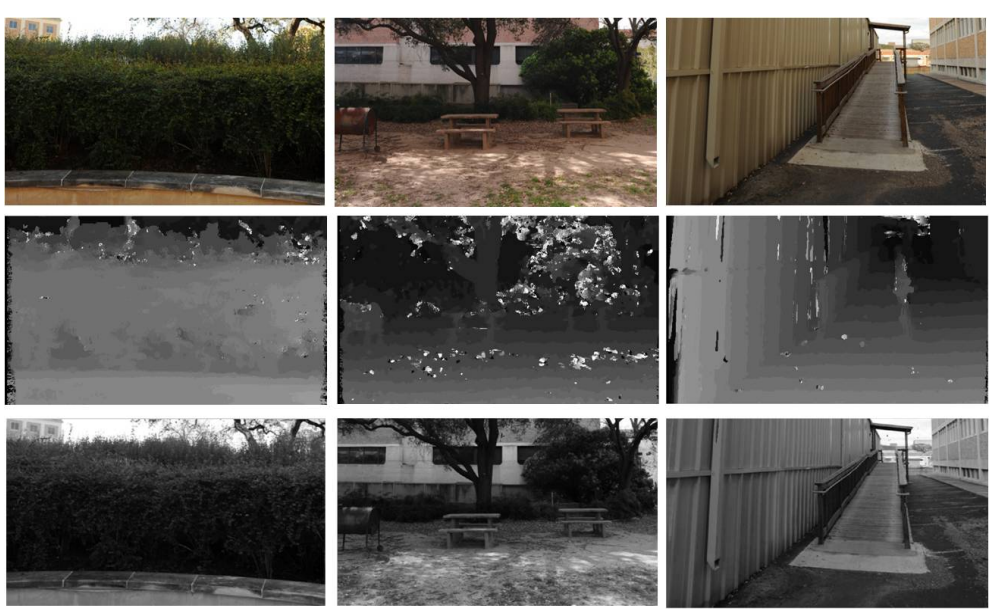

Fig. 3: The disparity and cyclopean images for stereoscopic images, the first row are left images from stereoscopic pairs, the second row is the disparity maps corresponding to the first row, and the last row are cyclopean maps.

Firstly, Gabor filter can be used as a simulation of binocular vision,as shown in Eq. 14.

$$
\begin{aligned}
& G\left(x, y, \sigma_{x}, \sigma_{y}, \xi_{x}, \xi_{y}, \theta\right) \\
& =\frac{1}{2 \pi \sigma_{x} \sigma_{y}} e^{-(1 / 2)\left[\left(R_{1} / \sigma_{x}\right)^{2}+\left(R_{2} / \sigma_{y}\right)^{2}\right]} e^{i\left(x \xi_{x}+x \xi_{y}\right)}
\end{aligned}
$$

Then we can get the cyclopean map by Eq. 15 and 16,as shown in Fig.3.

$$
\begin{gathered}
\omega_{l} E_{l}+\omega_{r} E_{r}=C \\
C l(x, y)=W_{L}(x, y) \times I_{L}(x, y)+W_{R}(x+d, y) \times I_{R}(x+d, y)
\end{gathered}
$$

In the process of stereo imaging, the deviation from the binocular angle will bring the occlusion. The pixel information in a visual angle may not be reflected in another perspective. Then in the process of estimating the disparity map, these pixels are considered to be irrelevant. Mathematical language to express it can be writen in Eq. 17,

$$
N C R_{e}(x, y)=\left\{\begin{array}{c}
1, \text { occluded } \\
0, \text { else }
\end{array}\right.
$$

Similarly, if two points of view can match the region, we think it is relevant in Eq. 18,

$$
C R_{e}(x, y)=\left\{\begin{array}{c}
1, \text { matched } \\
0, \text { else }
\end{array}\right.
$$




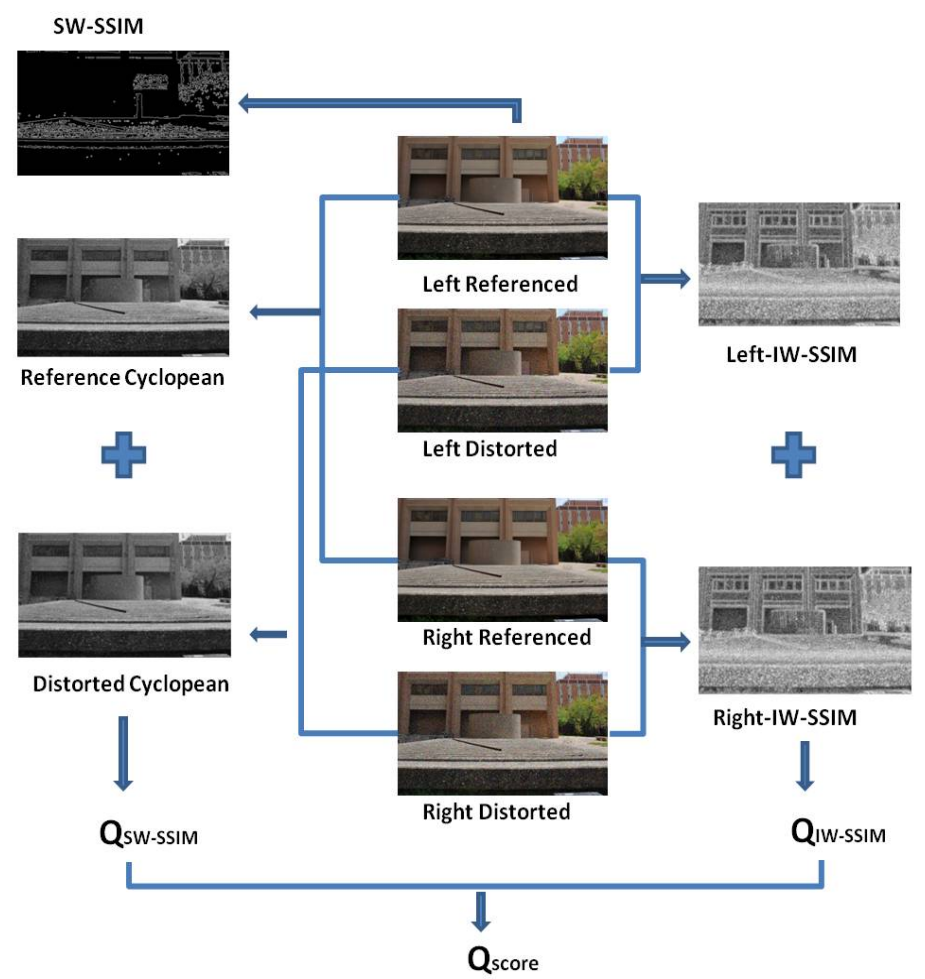

Fig. 4: Framework of the proposed virtual reality image quality assessment metric

\section{Proposed stereoscopic image quality assessment metric}

In this paper, we establish a set of accurate and effective evaluation for the virtual reality,as shown in Fig. 4. First, we divide the original reference and distorted image into two regions called binocular regions and monocular regions. then, the IW-SSIM or IW-PSNR values over the two regions are applied to certain weights. Finally, we pooling the stereoscopic images score by combing the two regions.

\subsection{Classification for binocular and monocular component}

In other people's research work, most of the disparity map is estimated using stereo matching algorithm, and the whole image is decomposed in the process. So, our task is to detect which areas of the image are correlated with the strong three-dimensional sense .

According to the second part of the paper. For stereo image quality evaluation, binocular region determines the three-dimensional sense, and the other part determine the quality of the 2D images. So we use the Hough transform 

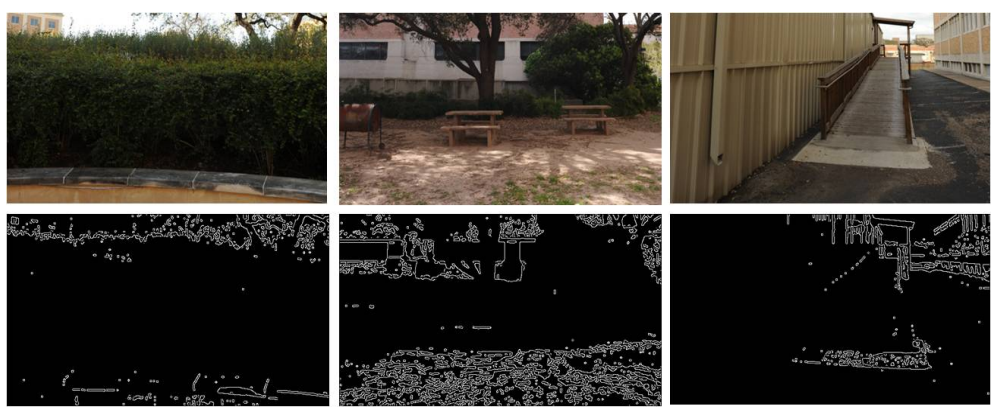

Fig. 5: Detection results of binocular information region of stereo images using Hough transform

to detect the edge information of the stereo image. On the basis of this, the classification for binouclar region is carried out $[21,23]$.

Hough transform is a feature extraction technology in image processing, it detects the object with a specific shape by a voting algorithm. Hough transform is put forward for the first time by Paul Hough. Promotion was made by Richard Duda and Peter Hart .The classical Hough transform for line detection in images and then Hough transform is extended to the recognition of objects of arbitrary shape, circle and ellipse, which can be used in this paper.

We know that a straight line in the Cartesian coordinate system can use $y=k x+b$ said, the main idea of Hough transform is exchanging the equation parameters and variables, $x, y$ as a known, and amount of $k, b$ as the coordinate variables.So line $y=k x+b$ in Cartesian coordinates in the parameter space said point $(k, b)$, and a $\left(x_{1}, y_{1}\right)$ point in Cartesian coordinates said as a straight line $y_{1}=k x_{1}+b$. The $(k, b)$ is an arbitrary point on the straight line. In order to facilitate the calculation, we will coordinate the parameter space representation for polar coordinates $\gamma$ and $\theta$. Because the line with a corresponding points $(\gamma, \theta)$ is the same, so it can be to find the image edge detection and image on each a non zero pixel point of. So it can be used to detect the line.

Using this method, we can easily divide the three-dimensional image . For different regions, we will use different calculation methods to give the weight of different sizes,as shown in Fig. 5.

\subsection{Content-weighted SSIM for monocular component}

Because the stereoscopic image is made up of the left and right visual angle, it is not complete to evaluate the visual information of a visual angle separately. However, many methods of plane image evaluation can still be used for reference. In this part, we consider the left and right angle of view as a plane image, and evaluate the weight of the information of the plane image, and obtain the overall evaluation results. This process fully reflects the three-dimensional im- 

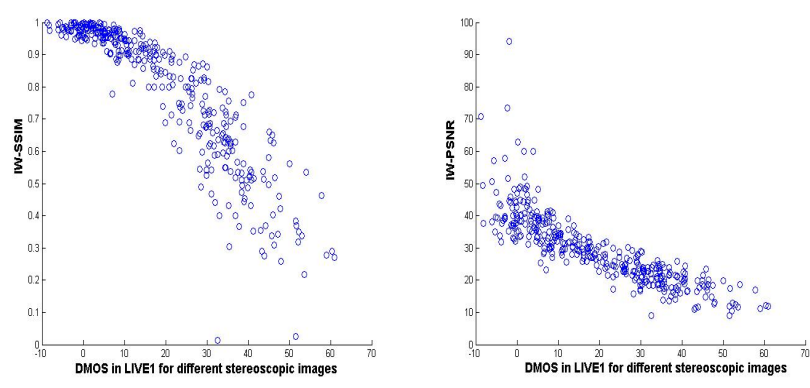

Fig. 6: The IW-SSIM and IW-PSNR score for stereoscopic iamges when we caculate left and right images As independent parts.

age of the inside, and the three-dimensional degree of irrelevant content of the performance.

By calculating, we can clearly see that the two methods can independently represent the quality of the image, as described in the previous section. So the validity of this method has been fully demonstrated when we evaluate the stereoscopic image to be divided into two parts in Eq. 19.

$$
Q_{I W S S I M}=\omega_{1} \cdot I W S S I M_{l e f t}+\omega_{2} \cdot I W S S I M_{\text {right }}
$$

In this part, we take $\omega_{1}=\omega_{2}=0.5$.The IW-SSIM and IW-PSNR scores for stereoscopic iamges can be compared in Fig. 6 .

\subsection{Stereo-weighted SSIM for cyclopean considering binocular vision}

As the content of the Hough transform can fully reflect the high frequency information in the stereoscopic image, it is proved by experiments that the information is very large. So in the use of Hough transform after the calibration area, need to be given a greater weight,we can describe it as Eq. 20.

The cyclopean maps contain the left and right image mutual information, which for three-dimensional evaluation is vital, so we also introduce cyclopean images, as a basis for further evaluation[16].

$$
Q_{S W S S I M}=\omega_{p}(x, y) \operatorname{IWSSIM}\left(C Y C_{\text {reference }}, C Y C_{\text {distortion }}\right)
$$

\subsection{Final predict}

Finally, $Q_{I W-S S I M}$ and $Q_{S W-S S I M}$ are combined into an overall quality score $Q$ in Eq. 21.

$$
Q=\omega \cdot Q_{I W S S I M}+(1-\omega) Q_{S W S S I M}
$$

After the weight search calculation, we get the value of $\omega$ is 0.6527 .In the next part of the test, we will also prove the rationality of this parameter. 


\section{Experimental results and analyses}

\subsection{Stereo image database}

In this paper, the process of establishment use the three-dimensional image database of University of Texas-Austin called 3D Image LIVE database[39], which can be divide into LIVEI and LIVEII. Quality validation of the proposed model can be convinced.

Stereo image database LIVEI contains 20 scenes from the different scenes. LIVEI contain 365 stereoscopic image pairs, and each image has its own subjective evaluation, the distortion of the image is symmetrical. A total of 5 types of distortion are included: JPEG, JP2K, WN, FF and Blur. The stereoscopic image pairs with different types of distortion can be used to defect the performance of detection algorithms in different situations[39].

Stereoscopic image database LIVEII contains 5 types of distortion types just like LIVEI, a total of 360 distortion stereo image pairs, each image corresponding to their respective subjective assessment value.

\subsection{Performance measures}

In this paper, four kinds of commonly used evaluation indexes are evaluated on the basis of two kinds of stereoscopic image database. The overall performance of the proposed model, that is, $P L C C$ (Pearsonlinear correlation coefficient), $S R O C C$ (Spearman rankorder correlation coefficient) , KROCC (Kendallrankorder correlation coefficient) and $R M S E$ (Root mean square error). The best performance corresponding to the $P L C C=S R O C C=K R O C C=1$ and $R S M E=0$.

At the same time, the 5 parameters[49] of the linear regression function is used in this paper,as Eq. 22

$$
\operatorname{DMOS}_{p}=\beta_{1}\left[\frac{1}{2}-\frac{1}{1+\exp \left(\beta_{2}\left(x-\beta_{3}\right)\right)}\right]+\beta_{4} x+\beta_{5}
$$

where $\beta_{1}, \beta_{2}, \beta_{3}, \beta_{4}$ and $\beta_{5}$,can be detemined by using the objective scores and subjective scores, then the experimental data can be caculated. The result can in this part can be seen in Table. 1 and 2 .

\subsection{Performance for different distortion types}

Theoretically speaking, for different types of distortion, stereoscopic image quality assessment should be able to solve the classification. But in the current research results, are from the overall point of view in the consideration of this issue. Evaluation of the index is also for the entire distortion of the threedimensional image library. So it is necessary for us to compare different types of distortion. In LIVEI, we compare the results obtained by different algorithms 
Table 1: Performance in LIVEI

\begin{tabular}{lllll}
\hline & PLCC & SROCC & KROCC & RSME \\
\hline FI-SSIM & 0.8699 & 0.8606 & 0.6659 & 8.0874 \\
FI-VIF & 0.9222 & 0.9188 & 0.7429 & 6.3423 \\
FI-UQI & 0.8996 & 0.9022 & 0.7207 & 7.1619 \\
Benoit & 0.8836 & 0.8854 & 0.6876 & 7.6784 \\
You & 0.9312 & $\mathbf{0 . 9 2 4 8}$ & 0.7503 & 5.9774 \\
Bensalma & 0.8887 & 0.8767 & 0.6780 & 7.5194 \\
Chen & 0.9181 & 0.9090 & 0.7256 & 6.5008 \\
Proposed & $\mathbf{0 . 9 3 2 2}$ & 0.9206 & $\mathbf{0 . 7 8 3 1}$ & $\mathbf{5 . 8 3 8 7}$ \\
\hline
\end{tabular}

Table 2: Performance in LIVEII

\begin{tabular}{lllll}
\hline & PLCC & SROCC & KROCC & RSME \\
\hline FI-SSIM & 0.6844 & 0.6795 & 0.5042 & 8.2295 \\
FI-VIF & 0.7234 & 0.7213 & 0.5525 & 7.7936 \\
FI-UQI & 0.7775 & 0.7720 & 0.5880 & 7.0990 \\
Benoit & 0.7642 & 0.7475 & 0.5553 & 7.2806 \\
You & 0.7744 & 0.7206 & 0.5361 & 7.1413 \\
Bensalma & 0.7699 & 0.7513 & 0.5761 & 7.2035 \\
Chen & 0.9065 & 0.9013 & 0.7307 & $\mathbf{4 . 7 6 6 5}$ \\
Proposed & $\mathbf{0 . 9 1 6 7}$ & $\mathbf{0 . 9 0 5 6}$ & $\mathbf{0 . 7 7 4 1}$ & 6.6437 \\
\hline
\end{tabular}

Table 3: PLCC on each individual distortion type in LIVEI

\begin{tabular}{llllll}
\hline & JEPG & JP2K & WN & GB & FF \\
\hline FI-SSIM & 0.2741 & 0.8210 & 0.9250 & 0.9080 & 0.7297 \\
FI-VIF & 0.6545 & 0.9412 & 0.9310 & 0.9573 & 0.7572 \\
FI-UQI & 0.7764 & $\mathbf{0 . 9 5 3 2}$ & 0.9374 & $\mathbf{0 . 9 6 3 3}$ & 0.7752 \\
Benoit & 0.5537 & 0.8789 & 0.9357 & 0.9181 & 0.7377 \\
You & 0.6287 & 0.9390 & 0.9344 & 0.9549 & $\mathbf{0 . 8 6 1 8}$ \\
Bensalma & 0.3868 & 0.8413 & 0.9157 & 0.9374 & 0.7385 \\
Chen & 0.5142 & 0.9006 & 0.9291 & 0.9387 & 0.7994 \\
Proposed & $\mathbf{0 . 7 8 5 6}$ & 0.9322 & $\mathbf{0 . 9 4 0 7}$ & 0.9453 & 0.8354 \\
\hline
\end{tabular}

on different distortion types. Since $P L C C$ is the most representative of the parameters in the above mentioned parameters, we are here to serve as the main basis for judging. The results of this part is shown in Table. 3 .

\subsection{Influence of the IW-SSIM and SW-SSIM}

As mentioned above, IW-SSIM is mainly used to evaluate the quality of the monocular part, while SW-SSIM is mainly used to evaluate the quality of the binocular part. So the contribution of the two parts is the content of our research. 
Table 4: Performance on each individual distortion type in LIVEI

\begin{tabular}{lllll}
\hline & PLCC & SROCC & KROCC & RSME \\
\hline IW-SSIM only & 0.9106 & 0.9031 & $\mathbf{0 . 7 9 3 9}$ & 6.7520 \\
SW-SSIM only & 0.8956 & 0.8864 & 0.7881 & 7.6545 \\
Proposed & $\mathbf{0 . 9 3 0 2}$ & $\mathbf{0 . 9 2 0 6}$ & 0.7831 & $\mathbf{5 . 8 3 8 7}$ \\
\hline
\end{tabular}

Table 5: Performance on each individual distortion type in LIVEII

\begin{tabular}{lllll}
\hline & PLCC & SROCC & KROCC & RSME \\
\hline IW-SSIM only & 0.8954 & 0.8861 & 0.7739 & $\mathbf{6 . 1 5 4 8}$ \\
SW-SSIM only & 0.8806 & 0.8753 & $\mathbf{0 . 7 9 8 1}$ & 6.9854 \\
Proposed & $\mathbf{0 . 9 1 6 7}$ & $\mathbf{0 . 9 0 5 6}$ & 0.7741 & 6.6437 \\
\hline
\end{tabular}

For using only IW-SSIM and using only the SW-SSIM section, we give the performance on the LIVEI and LIVEII libraries, respectively. As shown in Table. 4 and 5 .

\section{Conclusion}

Due to the three-dimensional images in virtual reality technology has an important position, and stereo images quality directly affect the whole system, so we make research on stereo image quality assessment in order to give the user a better virtual reality experience.

According to the stereoscopic images in virtual reality, this chapter firstly introduces the binocular stereo vision model, including the binocular model of stereo vision and the weight of single item information. Then, according to the $I W-S S I M$ and $S W-S S I M$ model, the objective evaluation model of stereoscopic image quality is put forward. This stereoscopic image quality assessment method effectively extends the previous stereo vision saliency model, and then combines the single channel stereo vision model to simulate the human eye stereo information fusion and processing process .After that, final quality evaluation model is set up. The above method effectively combines the characteristics of the human eye stereo vision with the weight of information, which not only greatly reduces the computational complexity, but also reduces the overall evaluation time.

The results show that the evaluation method can effectively evaluate the quality of the stereo image, and the correlation between the objective evaluation value and the subjective evaluation value is stronger. In the end, the stereoscopic vision mechanism of the human eye is the most important part for the virtual reality.However,This field has just started. Generally speaking, the eye stereoscopic vision information processing and three-dimensional information sensing mechanism is still in development stage. Multi-channel visual 
characteristics, contrast sensitivity visual characteristics and color visual characteristics, still need further research and analysis, which is significant in this field of research.

\section{Acknowledgements}

This research is partially supported by National Natural Science Foundation of China (No. 61471260 and No. 61271324), and Natural Science Foundation of Tianjin (No. 16JCYBJC16000).

\section{References}

1. Lin Y, Yang J, Lv Z, et al. A Self-Assessment Stereo Capture Model Applicable to the Internet of Things.[J]. Sensors, 2015, 15(8):20925-20944.

2. Lu F, Zhao Q, Yang G. A no-reference image quality assessment approach based on steerable pyramid decomposition using natural scene statistics[J]. Neural Computing and Applications, 2014, 26(1):77-90.

3. Yang J, Ru X, Lv Z, et al. Analysis of Camera Arrays Applicable to the Internet of Things:[J]. Sensors, 2016, 16(3).

4. Stern P. Virtual reality changes neuronal firing[J]. Science, 2014, 346(6213):1075-1075.

5. Komura T, Lau R W, Lin M C, et al. Virtual reality software and technology.[J]. IEEE Computer Graphics and Applications, 2015, 35(5):20-21.

6. Od K, Tanaka M, Yoshida A, et al. A video-rate stereo machine and its application to virtual reality[C]// IEEE Computer Vision and Pattern Recognition. 1999:18-20.

7. Isdale $\mathrm{J}$. What is virtual reality $[\mathrm{J}] .1998$.

8. Jack D, Boian R, Merians A, et al. Virtual Reality-Based Stroke Rehabilitation[C]// IEEE Trans. Neural Syst. Rehabil. Eng. 2015.

9. Zyda M. From visual simulation to virtual reality to games[J]. Computer, 2005, 38(9):2532 .

10. Sa A G D, Zachmann G. Integrating Virtual Reality for Virtual Prototyping[C]// Computers and Information in Engineering Conference. 1998:387-399.

11. Donalek C, Djorgovski S G, Cioc A, et al. Immersive and collaborative data visualization using virtual reality platforms[C]// IEEE International Conference on Big Data. IEEE Computer Society, 2014:609-614.

12. Staretu I. From Using Virtual Reality in the Training Process to, Virtual Engineering $[\mathrm{J}]$. Global Journal of Computer Science, 2014.

13. Hartanto D, Kampmann I L, Morina N, et al. Controlling social stress in virtual reality environments. PLoS ONE, 9(3), e92804[J]. Plos One, 2014, 9(3).

14. Liu Y, Yang J, Meng Q, et al. Stereoscopic image quality assessment method based on binocular combination saliency model[J]. Signal Processing, 2016, 125:237-248.

15. Yang J, Lin Y, Gao Z, et al. Quality Index for Stereoscopic Images by Separately Evaluating Adding and Subtracting[J]. Plos One, 2015, 10(12).

16. Wang Z, Li Q. Information content weighting for perceptual image quality assessment.[J]. IEEE Transactions on Image Processing A Publication of the IEEE Signal Processing Society, 2011, 20(5):1185-98.

17. Lin Y H, Wu J L. Quality assessment of stereoscopic 3D image compression by binocular integration behaviors.[J]. IEEE Transactions on Image Processing, 2014, 23(4):1527-42.

18. Benoit A, Callet P L, Campisi P, et al. Using disparity for quality assessment of stereoscopic images[C]// Image Processing, 2008. ICIP 2008. 15th IEEE International Conference on. IEEE, 2008:389-392.

19. Chen M J, Su C C, Kwon D K, et al. Full-reference quality assessment of stereopairs accounting for rivalry[J]. Signal Processing Image Communication, 2013, 28(9):1143-1155. 
20. You J, Wang X, Xing L, et al. Perceptual Quality Assessment for Stereoscopic Images Based on 2D Image Quality Metrics and Disparity Analysis[C]// International Workshop on Video Processing and Quality Metrics for Consumer Electronics. 2010.

21. Yang D, Guo P. Image modeling with combined optimization techniques for image semantic annotation[J]. Neural Computing and Applications, 2011, 20(7):1001-1015.

22. Zhang W, Qu C, Ma L, et al. Learning structure of stereoscopic image for no-reference quality assessment with convolutional neural network[J]. Pattern Recognition, 2016.

23. Chen L, Li C, Huang T, et al. Memristor crossbar-based unsupervised image learning $[\mathrm{J}]$. Neural Computing and Applications, 2014, 25(2):393-400.

24. Shao F, Lin W, Jiang G, et al. Models of Monocular and Binocular Visual Perception in Quality Assessment of Stereoscopic Images[J]. 2016, 2(2):1-1.

25. Damera-Venkata N, Kite T D, Geisler W S, et al. Image quality assessment based on a degradation model.[J]. IEEE Transactions on Image Processing A Publication of the IEEE Signal Processing Society, 2000, 9(4):636-650.

26. Wang Z, Bovik A C. A universal image quality index[J]. IEEE Signal Processing Letters, 2002, 9(3):81 - 84.

27. Wang Z, Bovik A C, Sheikh H R, et al. Image quality assessment: from error visibility to structural similarity[J]. IEEE Transactions on Image Processing, 2004, 13(4):600 - 612.

28. Wang Z, Simoncelli E P, Bovik A C. Multiscale structural similarity for image quality assessment[C]// Signals, Systems and Computers, 2003. Conference Record of the ThirtySeventh Asilomar Conference on. 2003:1398-1402 Vol.2.

29. Sheikh H R, Bovik A C, Veciana G D. An information fidelity criterion for image quality assessment using natural scene statistics[J]. IEEE Transactions on Image Processing A Publication of the IEEE Signal Processing Society, 2005, 14(12):2117-28.

30. Sheikh H R, Bovik A C. Image information and visual quality.[J]. IEEE Transactions on Image Processing A Publication of the IEEE Signal Processing Society, 2006, 15(2):430444 .

31. Chandler D M, Hemami S S. VSNR: A Wavelet-Based Visual Signal-to-Noise Ratio for Natural Images[J]. IEEE Transactions on Image Processing A Publication of the IEEE Signal Processing Society, 2007, 16(9):2284-98.

32. Wang Z, Li Q. Information content weighting for perceptual image quality assessment. J]. IEEE Transactions on Image Processing A Publication of the IEEE Signal Processing Society, 2011, 20(5):1185-98.

33. Lin Z, Zhang D, Mou X. RFSIM: A feature based image quality assessment metric using Riesz transforms[J]. 2010, 119(5):321-324.

34. Zhang L, Zhang L, Mou X, et al. FSIM: A Feature Similarity Index for Image Quality Assessment[J]. IEEE Transactions on Image Processing A Publication of the IEEE Signal Processing Society, 2011, 20(8):2378-86.

35. Liu A, Lin W, Narwaria M. Image quality assessment based on gradient similarity. $[\mathrm{J}]$. IEEE Transactions on Image Processing A Publication of the IEEE Signal Processing Society, 2012, 21(4):1500-12.

36. Zhang L, Shen Y, Li H. VSI: a visual saliency-induced index for perceptual image quality assessment.[J]. IEEE Transactions on Image Processing, 2014, 23(10):4270-81.

37. Larson E C, Chandler D M. Most apparent distortion: full-reference image quality assessment and the role of strategy[J]. Journal of Electronic Imaging, 2010, 19(1):143-153.

38. Mittal A, Moorthy A K, Bovik A C. No-reference image quality assessment in the spatial domain. $[\mathrm{J}]$. IEEE Transactions on Image Processing A Publication of the IEEE Signal Processing Society, 2012, 21(12):4695-4708.

39. Moorthy A K, Su C C, Mittal A, et al. Subjective evaluation of stereoscopic image quality[J]. Signal Processing Image Communication, 2013, 28(8):870-883.

40. Ye P, Doermann D. No-reference image quality assessment based on visual codebook[C]// IEEE International Conference on Image Processing. 2011:3089-3092.

41. Doermann D. Unsupervised feature learning framework for no-reference image quality assessment[C]// IEEE Conference on Computer Vision and Pattern Recognition. IEEE Computer Society, 2012:1098-1105.

42. Li C, Bovik A C, Wu X. Blind image quality assessment using a general regression neural network.[J]. IEEE Transactions on Neural Networks, 2011, 22(5):793-799.

43. Kang L, Ye P, Li Y, et al. Convolutional Neural Networks for No-Reference Image Quality Assessment[J]. 2014:1733-1740. 
44. Moorthy A K, Bovik A C. Blind Image Quality Assessment: From Natural Scene Statistics to Perceptual Quality[J]. IEEE Transactions on Image Processing A Publication of the IEEE Signal Processing Society, 2011, 20(12):3350-64.

45. Saad M A, Bovik A C, Charrier C. Blind Image Quality Assessment: A Natural Scene Statistics Approach in the DCT Domain[J]. IEEE Transactions on Image Processing A Publication of the IEEE Signal Processing Society, 2012, 21(8):3339-52.

46. Tang H, Joshi N, Kapoor A. Blind Image Quality Assessment Using Semi-supervised Rectifier Networks[C]// IEEE Conference on Computer Vision and Pattern Recognition. 2014:2877-2884

47. Xue W, Zhang L, Mou X. Learning without Human Scores for Blind Image Quality Assessment[J]. 2013, 9(4):995-1002.

48. Li C, Bovik A C. Content-weighted video quality assessment using a three-component image model[J]. Journal of Electronic Imaging, 2010, 29(1):143-153.

49. Gottschalk P G, Dunn J R. The five-parameter logistic: A characterization and comparison with the four-parameter logistic [J]. Analytical Biochemistry, 2005, 343(1):54-65. 